\title{
Antibiotic Prescribing for Acute Bronchitis: How Low Can We Go?
}

By the time I graduated from medical school in 1975, I had learned that most respiratory tract infections in otherwise healthy children and adults were caused by viruses, such as parainfluenza, influenza, adenovirus, and respiratory syncytial virus. This learning was reinforced during my family practice residency training in Charleston, SC. I was taught that the challenge of primary care practice is to distinguish the many patients with benign, selflimited respiratory tract infections from those patients who are more seriously ill with bacterial pneumonia and who need an antibiotic to recover more quickly and more certainly. Armed with this scientific knowledge, I marched valiantly into practice, determined to base my prescribing on good science. It was only in rural practice that I clearly recall regularly confronting syndromes called "acute bronchitis" and "sinusitis," for which patients seemed to expect an antibiotic and for which their previous physicians had prescribed one. "I get this bronchitis every fall. Dr. Smith gives me a shot of penicillin and I'm fine 2 days later." With modest success I was able to talk patients out of this approach, but I more frequently failed than succeeded, though many were willing to take my prescription for pills as an acceptable alternative to the shot in the buttock.

A quarter-century later I am still fighting the good battle of appropriate antibiotic use for respiratory tract infections. After a 25-year cold war, however, the battle is heating up! The Centers for Disease Control and Prevention has entered the fray, urging us and our patients to use antibiotics sparingly and wisely for acute respiratory tract infections. ${ }^{1}$ Family physicians have been named as a major contributor to the problem of bacterial resistance, and the reasons we participate in the problem have been thoroughly described. In the words

Submitted, 14 August 2000.

From the Department of Family Medicine, Michigan State University College of Human Medicine, East Lansing. Address reprint requests to John $M$. Hickner, MD, MS, Department of Family Practice, Michigan State University College of Human Medicine, B 111 Clinical Center, Michigan State University, East Lansing, MI 48824. of that wise philosopher Pogo, "We have seen the enemy, and he is us."

No doubt, we can do better. Several investigators have described successful methods of reducing antibiotic use for respiratory tract infections. Gonzales et $\mathrm{al}^{2}$ found that combined patient and clinician education was effective in reducing antibiotic use for acute bronchitis from $74 \%$ to $48 \%$ in a health maintenance organization setting. Using a quality improvement approach and a computerbased patient record in an academic family practice setting, Ornstein and colleagues ${ }^{3}$ reduced antibiotic prescribing for acute bronchitis from $60 \%$ to less than $30 \%$.

These and other initiatives show that it is possible to reduce use of antibiotics for acute respiratory tract infections, but how low can we go? The report by Hueston et $\mathrm{al}^{4}$ in this issue of the $\mathcal{F} A B F P$ suggests that, for patients with the diagnosis of acute bronchitis, the answer might not be $0 \%$. After the successful campaign to reduce antibiotic use for acute bronchitis by Ornstein et al, ${ }^{3}$ a stubborn residual of $26 \%$ of patients received an antibiotic. Why? To answer this question, Hueston and colleagues compared clinical characteristics of patients who received an antibiotic with those who did not in a retrospective chart review conducted after the largely successful intervention. They compared clinician characteristics as well. The investigators nearly came up empty handed. Patients' signs and symptoms were poor predictors of antibiotic prescribing in these recalcitrant cases. There were no clinician factors that predicted antibiotic prescribing-no "resistant" physicians were found. The study was, however, underpowered to detect some potentially important clinical predictors. For example, an antibiotic was prescribed for $36 \%$ of patients with fever, $42 \%$ of patients with shortness of breath, $56 \%$ of patients with chest pain, and $75 \%$ of patients with sweats, though a small proportion of patients had these complaints. Presence of purulent sputum, a common reason for antibiotic prescribing, was not assessed.

Children were much less likely to receive an antibiotic than adults, $3 \%$ compared with $34 \%$. Patient expectation could not be measured in this 
study, but it is a likely explanation for this striking difference. Perhaps parents are more willing to take a watch-and-wait approach for their children because the current national antiantibiotic campaign has begun to have some effect, whereas adults who might have received an antibiotic for a similar infection in the past are more likely to demand an antibiotic. Recently, Dosh and colleagues ${ }^{5}$ found that patient demand for an antibiotic for an upper respiratory tract infection, acute bronchitis, and acute sinusitis was strongly predicted by having received an antibiotic for a previous similar illness.

I doubt that patient expectation is the only factor driving continued antibiotic prescribing for acute bronchitis as well as other common acute respiratory tract infections, or ARIs. (I prefer this terminology rather than the anatomic nomenclature of bronchitis, sinusitis, and upper respiratory tract infection, because the same organism might cause acute bronchitis in one person, simple upper respiratory tract infection in another, and acute sinusitis in yet another.) Based on my many discussions with family physicians and from our own research, physicians do believe that some patients with ARIs benefit from antibiotic treatment. In a prospective descriptive study of diagnosis and treatment of ARIs in a rural primary care research network, we found a typical pattern of antibiotic prescribing: $21 \%$ for upper respiratory tract infections, $80 \%$ for acute bronchitis, and $98 \%$ for acute sinusitis. ${ }^{5}$ In this "untrained" group of physicians and patients, antibiotic prescribing was not independently predicted by any symptoms but was predicted by a small number of physical findings: discolored nasal discharge, rales or rhonchi, sinus tenderness, and postnasal drainage. Surprisingly, patient expectation was not an independent predictor of antibiotic prescribing.

Hueston and colleagues speculate that factors other than signs, symptoms, and clinician characteristics predict antibiotic prescribing for acute bronchitis. I agree. In our study of ARIs, we explored this issue by asking clinicians the following question for each patient for whom an antibiotic was prescribed: Other than the diagnosis, what other factors did you consider when prescribing an antibiotic for this patient? Clinicians reported other factors in $95 \%$ of cases. The most common reasons were patient not improving $(21 \%)$, patient getting worse $(19 \%)$, and patient has been sick for too long $(19 \%)$. Clinicians prescribed an antibiotic in $98 \%$ of cases in which they believed the likelihood of an adverse outcome was high if no antibiotic was prescribed.

How low can we go with antibiotic prescribing for acute respiratory infections? Here are my targets for the year 2005: upper respiratory tract infections $10 \%$; acute bronchitis $25 \%$; acute sinusitis $50 \%$. Until the prognostic uncertainty of prolonged symptoms is resolved (or not!) by a large randomized clinical trial, many physicians will continue to reach for the prescription pad for patients who have acute respiratory tract infections who have been sick for too long or who are not getting better.

\section{John M. Hickner, MD, MS} East Lansing, Mich

\section{References}

1. Schwartz B, Bell DM, Hughes JM. Preventing the emergence of antimicrobial resistance: a call for action by clinicians, public health officials, and patients. JAMA 1997;278:944-5.

2. Gonzales R, Steiner JF, Lum A, Barrett PH Jr. Decreasing antibiotic use in ambulatory practice: impact of a multidimensional intervention on the treatment of uncomplicated acute bronchitis in adults.. JAMA 1999;281:1512-9.

3. Ornstein SM, Jenkins RG, Lee FW, et. al. The computer-based patient record as a CQI tool in a family medicine center. Jt Comm J Qual Improv 1997;23:347-61.

4. Hueston WJ, Hopper JE, Dacus EN, Mainous AG, Roetzhein RG. Why are antibiotics prescribed for patients with acute bronchitis? A postintervention analysis. J Am Board Fam Pract 2000;13:398-402.

5. Dosh S, Hickner JM, Mainous AG 3rd, Ebell $M$. Predictors of antibiotic prescribing for nonspecific upper respiratory infections, acute bronchitis, and acute sinusitis: an UPRNet study. Upper Peninsula Research Network. J Fam Pract 2000;49:407-14. 